\title{
COMPLETE MONOTONICITY OF A FUNCTION INVOLVING THE DIVIDED DIFFERENCE OF PSI FUNCTIONS
}

\author{
FENG QI ${ }^{凶}$, PIETRO CERONE and SEVER S. DRAGOMIR
}

(Received 30 August 2012; accepted 9 October 2012; first published online 18 January 2013)

\begin{abstract}
Necessary and sufficient conditions are presented for a function involving the divided difference of the psi function to be completely monotonic and for a function involving the ratio of two gamma functions to be logarithmically completely monotonic. From these, some double inequalities are derived for bounding polygamma functions, divided differences of polygamma functions, and the ratio of two gamma functions.

2010 Mathematics subject classification: primary 26A48, 33B15; secondary 26A51, 26 D15.

Keywords and phrases: necessary and sufficient condition, complete monotonicity, logarithmically complete monotonicity, divided difference, ratio of two gamma functions, inequality, psi function, polygamma function.
\end{abstract}

\section{Introduction}

A function $f$ is called completely monotonic on an interval $I \subseteq \mathbb{R}$ if $f$ has derivatives of all orders on $I$ and

$$
(-1)^{k} f^{(k)}(x) \geq 0
$$

holds for all $k \geq 0$ on $I$. For convenience, in what follows, the class of completely monotonic functions on $I$ is denoted by $C[I]$. The class of completely monotonic functions may be characterised by [14, p. 161, Theorem 12b] which reads that a necessary and sufficient condition for $f(x)$ to be completely monotonic for $0<x<\infty$ is that

$$
f(x)=\int_{0}^{\infty} e^{-x t} d \alpha(t),
$$

where $\alpha(t)$ is nondecreasing and the integral converges for $0<x<\infty$. This means that $f \in C[(0, \infty)]$ if and only if $f$ is a Laplace transform of the measure $\mu$.

A function $f$ is said to be logarithmically completely monotonic on an interval $I \subseteq \mathbb{R}$ if it has derivatives of all orders on $I$ and its $\log$ arithm $\log f$ satisfies

$$
(-1)^{k}(\log f(x))^{(k)} \geq 0
$$

(C) 2013 Australian Mathematical Publishing Association Inc. 0004-9727/2013 \$16.00 
for $k \in \mathbb{N}$ on $I$. In what follows, the set of all logarithmically completely monotonic functions on $I$ will be denoted by $\mathcal{L}[I]$. The logarithmically completely monotonic functions on $(0, \infty)$ are characterised in [1] as the infinitely divisible completely monotonic functions studied in [4].

The inclusive relationship $\mathcal{L}[I] \subset C[I]$ has been proved in several papers. For detailed information, see [7, Section 1.5], [9, Section 1.3], [10, Section 1] and closely related references therein. Furthermore, it was discovered in [1] that every Stieltjes transform belongs to $\mathcal{L}[(0, \infty)]$, where a function $f$ defined on $(0, \infty)$ is called a Stieltjes transform if it can be expressed in the form

$$
f(x)=a+\int_{0}^{\infty} \frac{1}{s+x} d \mu(s)
$$

for some nonnegative number $a$ and some nonnegative measure $\mu$ on $[0, \infty)$ satisfying $\int_{0}^{\infty} d \mu(s) /(1+s)<\infty$. For more information on this topic, see [11].

The classical Euler gamma function may be defined for $x>0$ by

$$
\Gamma(x)=\int_{0}^{\infty} t^{x-1} e^{-t} d t .
$$

The logarithmic derivative of $\Gamma(x)$, denoted by $\psi(x)=\Gamma^{\prime}(x) / \Gamma(x)$, is called the psi function, and $\psi^{(k)}(x)$ for $k \in \mathbb{N}$ are called the polygamma functions. The special functions $\Gamma(x), \psi(x)$ and $\psi^{(k)}(x)$ for $k \in \mathbb{N}$ are fundamental and important and have many applications in mathematical sciences.

In [6, Thorem 1.3], the following necessary and sufficient conditions were established: the function

$$
\psi(x)-\log x+\frac{\alpha}{x}
$$

belongs to $C[(0, \infty)]$ if and only if $\alpha \geq 1$, as does the negative of (1.2) if and only if $\alpha \leq \frac{1}{2}$. For more information on equivalences of these necessary and sufficient conditions, see [2, 5], [8, pp. 1977-1978, Section 1.5], and the review articles [7, 9] and the many references cited therein.

In order to alternatively verify the monotonicity and convexity of the function

$$
\left(\frac{\Gamma(x+t)}{\Gamma(x+s)}\right)^{1 /(t-s)}-x
$$

for $x \in(-\alpha, \infty)$, where $s$ and $t$ are real numbers and $\alpha=\min \{s, t\}$, the following complete monotonicity of the divided difference of the psi functions was discovered in [5, 8]: for real numbers $s$ and $t$ and $\alpha=\min \{s, t\}$, the function

$$
\delta_{s, t}(x)= \begin{cases}\frac{\psi(x+t)-\psi(x+s)}{t-s}-\frac{2 x+s+t+1}{2(x+s)(x+t)}, & s \neq t \\ \psi^{\prime}(x+s)-\frac{1}{x+s}-\frac{1}{2(x+s)^{2}}, & s=t\end{cases}
$$


for $|t-s|<1$ and its negative $-\delta_{s, t}(x)$ for $|t-s|>1$ belong to $C[(-\alpha, \infty)]$. For the history, background, and recent developments of the study of the function (1.3), see $[3,8]$, [7, Sections 3.9, 3.20.1 and 6.1] and closely related references therein.

We now generalise the function $\delta_{s, t}(x)$ in (1.4) by introducing a parameter $\lambda$ as follows. For real numbers $s$ and $t$, define

$$
\delta_{s, t ; \lambda}(x)= \begin{cases}\frac{\psi(x+t)-\psi(x+s)}{t-s}-\frac{2 x+s+t+2 \lambda}{2(x+s)(x+t)}, & s \neq t \\ \psi^{\prime}(x+s)-\frac{1}{x+s}-\frac{\lambda}{(x+s)^{2}}, & s=t\end{cases}
$$

on $(-\alpha, \infty)$, where $\lambda \in \mathbb{R}$ and $\alpha=\min \{s, t\}$. It is clear from (1.4) and (1.5) that $\delta_{s, t ; 1 / 2}(x)=\delta_{s, t}(x)$.

Motivated both by the necessary and sufficient conditions for the function (1.2) to belong to $C[(0, \infty)]$ and by the complete monotonicity of the function (1.4), it is natural to ask: what are the necessary and sufficient conditions such that the function (1.5) belongs to $C[(-\alpha, \infty)]$ ? This question is answered by our Theorem 1.1 below.

THEOREM 1.1. Let $s$ and $t$ be real numbers and let $\alpha=\min \{s, t\}$.

(1) For $|t-s|<1$ :

(a) $\delta_{s, t ; \lambda}(x) \in C[(-\alpha, \infty)]$ if and only if $\lambda \leq \frac{1}{2}$;

(b) $-\delta_{s, t ; \lambda}(x) \in C[(-\alpha, \infty)]$ if and only if $\lambda \geq 1-\frac{1}{2}|t-s|$.

(2) $\operatorname{For}|t-s|>1$ :

(a) $\delta_{s, t ; \lambda}(x) \in C[(-\alpha, \infty)]$ if and only if $\lambda \leq 1-\frac{1}{2}|t-s|$;

(b) $-\delta_{s, t ; \lambda}(x) \in C[(-\alpha, \infty)]$ if and only if $\lambda \geq \frac{1}{2}$.

(3) For $|t-s|=1$ :

(a) $\delta_{s, t ; \lambda}(x) \in C[(-\alpha, \infty)]$ if and only if $\lambda<\frac{1}{2}$;

(b) $-\delta_{s, t ; \lambda}(x) \in C[(-\alpha, \infty)]$ if and only if $\lambda>\frac{1}{2}$;

(c) the function $\delta_{s, t ; \lambda}(x)$ is identically zero if and only if $\lambda=\frac{1}{2}$.

As a direct application of Theorem 1.1, the logarithmically complete monotonicity results of a function involving the ratio of two gamma functions can be deduced as follows.

THEOREM 1.2. For real numbers $s$ and $t$, define

$$
H_{s, t ; \lambda}(x)= \begin{cases}\frac{(x+t)^{\lambda /(t-s)-1 / 2}}{(x+s)^{\lambda /(t-s)+1 / 2}\left(\frac{\Gamma(x+t)}{\Gamma(x+s)}\right)^{1 /(t-s)},} & s \neq t \\ \frac{1}{x+t} \exp \left(\psi(x+t)+\frac{\lambda}{x+t}\right), & s=t\end{cases}
$$

on $(-\alpha, \infty)$, where $\lambda \in \mathbb{R}$ and $\alpha=\min \{s, t\}$. The following conclusions are valid.

(1) For $|t-s|<1$ :

(a) $H_{s, t ; \lambda}(x) \in \mathcal{L}[(-\alpha, \infty)]$ if and only if $\lambda \geq 1-\frac{1}{2}|t-s|$;

(b) $\quad\left(H_{s, t ; \lambda}(x)\right)^{-1} \in \mathcal{L}[(-\alpha, \infty)]$ if and only if $\lambda \leq \frac{1}{2}$. 
(2) For $|t-s|>1$ :

(a) $H_{s, t ; \lambda}(x) \in \mathcal{L}[(-\alpha, \infty)]$ if and only if $\lambda \geq \frac{1}{2}$;

(b) $\quad\left(H_{s, t ; \lambda}(x)\right)^{-1} \in \mathcal{L}[(-\alpha, \infty)]$ if and only if $\lambda \leq 1-\frac{1}{2}|t-s|$.

(3) For $|t-s|=1$ :

(a) $H_{s, t ; \lambda}(x) \in \mathcal{L}[(-\alpha, \infty)]$ if and only if $\lambda>\frac{1}{2}$;

(b) $\left(H_{s, t ; \lambda}(x)\right)^{-1} \in \mathcal{L}[(-\alpha, \infty)]$ if and only if $\lambda<\frac{1}{2}$;

(c) the function $H_{s, t ; \lambda}(x)$ identically equals 1 on $(-\alpha, \infty)$ if and only if $\lambda=\frac{1}{2}$.

As consequences of Theorems 1.1 and 1.2, the following double inequalities are immediately derived for the polygamma functions, the divided differences of polygamma functions, and the ratio of two gamma functions.

THeOREM 1.3. The following statements are true.

(1) For $x>0$, the double inequality

$$
\beta_{1} \frac{k !}{x^{k+1}}<(-1)^{k+1} \psi^{(k)}(x)-\frac{(k-1) !}{x^{k}}<\gamma_{1} \frac{k !}{x^{k+1}}
$$

holds if and only if $\beta_{1} \leq \frac{1}{2}$ and $\gamma_{1} \geq 1$.

(2) Let $a$ and $b$ be positive numbers and $k \in \mathbb{N}$.

(a) For $0<|b-a|<1$, the double inequality

$$
\begin{aligned}
\frac{(k-1) !}{2} & \left(\frac{1}{a^{k}}+\frac{1}{b^{k}}\right)+\beta_{2} \frac{(k-1) !}{b-a}\left(\frac{1}{a^{k}}-\frac{1}{b^{k}}\right) \\
< & \frac{(-1)^{k-1}\left(\psi^{(k-1)}(b)-\psi^{(k-1)}(a)\right)}{b-a} \\
< & \frac{(k-1) !}{2}\left(\frac{1}{a^{k}}+\frac{1}{b^{k}}\right)+\gamma_{2} \frac{(k-1) !}{b-a}\left(\frac{1}{a^{k}}-\frac{1}{b^{k}}\right)
\end{aligned}
$$

holds if and only if $\beta_{2} \leq \frac{1}{2}$ and $\gamma_{2} \geq 1-\frac{1}{2}|b-a|$.

(b) For $|b-a|>1$, the double inequality (1.7) is reversed if and only if $\beta_{2} \leq$ $1-\frac{1}{2}|b-a|$ and $\gamma_{2} \geq \frac{1}{2}$.

(3) Let $a$ and $b$ be positive numbers and $k \in \mathbb{N}$.

(a) For $0<|b-a|<1$, the double inequality

$$
\frac{a^{\beta_{3} /(b-a)+1 / 2}}{b^{\beta_{3} /(b-a)-1 / 2}}<\left(\frac{\Gamma(b)}{\Gamma(a)}\right)^{1 /(b-a)}<\frac{a^{\gamma_{3} /(b-a)+1 / 2}}{b^{\gamma_{3} /(b-a)-1 / 2}}
$$

holds if and only if $\beta_{3} \geq 1-\frac{1}{2}|b-a|$ and $\gamma_{3} \leq \frac{1}{2}$.

(b) For $|b-a|>1$, the double inequality (1.8) is reversed if and only if $\beta_{3} \leq$ $1-\frac{1}{2}|b-a|$ and $\gamma_{3} \geq \frac{1}{2}$. 
(4) Let $s$ and t be real numbers, $\alpha=\min \{s, t\}$, and $x \in(\rho, \infty) \subset(-\alpha, \infty)$.

(a) For $0<|t-s|<1$, the double inequality

$$
\begin{aligned}
& \frac{(\rho+t)^{\beta_{4} /(t-s)-1 / 2}}{(\rho+s)^{\beta_{4} /(t-s)+1 / 2}}\left(\frac{\Gamma(\rho+t)}{\Gamma(\rho+s)}\right)^{1 /(t-s)} \frac{(x+s)^{\beta_{4} /(t-s)+1 / 2}}{(x+t)^{\beta_{4} /(t-s)-1 / 2}} \\
& \quad<\left(\frac{\Gamma(x+t)}{\Gamma(x+s)}\right)^{1 /(t-s)} \\
& \quad<\frac{(\rho+t)^{\gamma_{4} /(t-s)-1 / 2}}{(\rho+s)^{\gamma_{4} /(t-s)+1 / 2}}\left(\frac{\Gamma(\rho+t)}{\Gamma(\rho+s)}\right)^{1 /(t-s)} \frac{(x+s)^{\gamma_{4} /(t-s)+1 / 2}}{(x+t)^{\gamma_{4} /(t-s)-1 / 2}}
\end{aligned}
$$

holds if and only if $\beta_{4} \leq \frac{1}{2}$ and $\gamma_{4} \geq 1-\frac{1}{2}|t-s|$.

(b) For $|t-s|>1$, the inequality (1.9) is reversed if and only if $\beta_{4} \geq \frac{1}{2}$ and $\gamma_{4} \leq 1-\frac{1}{2}|t-s|$.

REMARK 1.4. We remark that taking $a=x+\frac{1}{2}$ and $b=x+1$ in the right-hand side of (1.8) yields

$$
\left(\frac{\Gamma(x+1)}{\Gamma\left(x+\frac{1}{2}\right)}\right)^{2}<\frac{\left(x+\frac{1}{2}\right)^{3 / 2}}{(x+1)^{1 / 2}}=\left(1-\frac{1}{2(x+1)}\right)^{1 / 2}\left(x+\frac{1}{2}\right)
$$

on $\left(-\frac{1}{2}, \infty\right)$, which is obviously better than the inequality

$$
\left(\frac{\Gamma(x+1)}{\Gamma\left(x+\frac{1}{2}\right)}\right)^{2}<x+\frac{1}{2}
$$

on $\left(-\frac{1}{2}, \infty\right)$. The inequality $(1.10)$ is a long-standing upper bound obtained in [12]. For more information about the inequality (1.10), see [7, pp. 21-22, Section 3.1].

REMARK 1.5. There have been some results similar to but different from our Theorems 1.2 and 1.3. For details, see [10] or related contents in [7, 9].

\section{Proofs of theorems}

We are now in a position to prove our theorems.

Proof of Theorem 1.1. For $|s-t|=1$, by (1.5), we may equivalently discuss the complete monotonicity of the function

$$
\delta_{s, s+1 ; \lambda}(x)=\frac{1-2 \lambda}{2(x+s)(x+s+1)},
$$

which follows obviously from the fact that the product of finitely many completely monotonic functions is still completely monotonic.

For $s=t$, we may equivalently discuss the complete monotonicity of the function

$$
\psi^{\prime}(x)-\frac{1}{x}-\frac{\lambda}{x^{2}}
$$

on $(0, \infty)$, which can be derived directly from [2, Theorem 2] and [6, Thorem 1.3] mentioned above. 
For $s \neq t$ and $s-t \neq \pm 1$, the function $\delta_{s, t ; \lambda}(x)$ can be rewritten as

$$
\delta_{s, t ; \lambda}(x)=\frac{1}{t-s} \int_{s}^{t} \psi^{\prime}(x+u) d u-\frac{1}{2}\left(\left(1-\frac{2 \lambda}{t-s}\right) \frac{1}{x+t}+\left(1+\frac{2 \lambda}{t-s}\right) \frac{1}{x+s}\right) .
$$

Differentiating consecutively and employing the well-known formulas

$$
\psi^{(k)}(x)=(-1)^{k+1} \int_{0}^{\infty} \frac{t^{k}}{1-e^{-t}} e^{-x t} d t
$$

and

$$
\frac{1}{x^{r}}=\frac{1}{\Gamma(r)} \int_{0}^{\infty} t^{r-1} e^{-x t} d t
$$

for $k \in \mathbb{N}$ and positive numbers $x>0$ and $r>0$, we obtain

$$
\begin{aligned}
\delta_{s, t ; \lambda}^{(k)}(x)= & \frac{1}{t-s} \int_{s}^{t} \psi^{(k+1)}(x+u) d u \\
& -\frac{(-1)^{k} k !}{2}\left(\left(1-\frac{2 \lambda}{t-s}\right) \frac{1}{(x+t)^{k+1}}+\left(1+\frac{2 \lambda}{t-s}\right) \frac{1}{(x+s)^{k+1}}\right) \\
= & \frac{(-1)^{k}}{t-s} \int_{s}^{t} \int_{0}^{\infty} \frac{v^{k+1}}{1-e^{-v}} e^{-(x+u) v} d v d u \\
& -\frac{(-1)^{k}}{2}\left(\left(1-\frac{2 \lambda}{t-s}\right) \int_{0}^{\infty} v^{k} e^{-(x+t) v} d v+\left(1+\frac{2 \lambda}{t-s}\right) \int_{0}^{\infty} v^{k} e^{-(x+s) v} d v\right) \\
= & (-1)^{k} \int_{0}^{\infty}\left(\frac{1}{t-s} \int_{s}^{t} \frac{v}{1-e^{-v}} e^{-u v} d u\right. \\
& \left.-\frac{1}{2}\left(\left(1-\frac{2 \lambda}{t-s}\right) e^{-t v}+\left(1+\frac{2 \lambda}{t-s}\right) e^{-s v}\right)\right) v^{k} e^{-x v} d v \\
= & (-1)^{k} \int_{0}^{\infty}\left(\frac{e^{-s v}-e^{-t v}}{(t-s)\left(1-e^{-v}\right)}-\frac{e^{-s v}+e^{-t v}}{2}-\frac{\lambda\left(e^{-s v}-e^{-t v}\right)}{t-s}\right) v^{k} e^{-x v} d v \\
= & (-1)^{k} \int_{0}^{\infty}\left(\left(\frac{1}{1-e^{-v}}-\lambda\right) \frac{e^{-s v}-e^{-t v}}{t-s}-\frac{e^{-s v}+e^{-t v}}{2}\right) v^{k} e^{-x v} d v \\
= & (-1)^{k} \int_{0}^{\infty}\left(\frac{1}{1-e^{-v}}-\lambda-\frac{(t-s)\left(e^{-s v}+e^{-t v}\right)}{2\left(e^{-s v}-e^{-t v}\right)}\right) \frac{e^{-s v}-e^{-t v}}{t-s} v^{k} e^{-x v} d v \\
= & (-1)^{k} \int_{0}^{\infty}\left(\frac{1}{1-e^{-v}}-\frac{e^{-s v}-e^{-t v}}{2 \tanh ((t-s) v / 2)}-\lambda\right) \frac{v^{k} e^{-x v} d v}{t-s}
\end{aligned}
$$

for $k \in\{0\} \cup \mathbb{N}$. Therefore, if

$$
\begin{aligned}
\lambda & \leq \frac{1}{1-e^{-v}}-\frac{t-s}{2 \tanh ((t-s) v / 2)} \\
& =\frac{2 e^{v} \tanh ((t-s) v / 2)-(t-s)\left(e^{v}-1\right)}{2\left(e^{v}-1\right) \tanh ((t-s) v / 2)} \triangleq \lambda(v, t-s)
\end{aligned}
$$


for all $v \in(0, \infty)$, then

$$
(-1)^{k} \delta_{s, t ; \lambda}^{(k)}(x) \geq 0 \quad \text { and } \quad \delta_{s, t ; \lambda}(x) \in C[(-\alpha, \infty)] ;
$$

if the inequality (2.1) reverses, then

$$
(-1)^{k} \delta_{s, t ; \lambda}^{(k)}(x) \leq 0 \quad \text { and } \quad-\delta_{s, t ; \lambda}(x) \in C[(-\alpha, \infty)] .
$$

Straightforward computation gives

$$
\frac{\partial \lambda(v, r)}{\partial v}=\frac{1}{4} r^{2} \operatorname{csch}^{2}\left(\frac{r v}{2}\right)-\frac{e^{v}}{\left(e^{v}-1\right)^{2}}=\frac{1}{v^{2}}\left(\left(\frac{r v}{2}\right)^{2} \operatorname{csch}^{2}\left(\frac{r v}{2}\right)-\left(\frac{v}{2}\right)^{2} \operatorname{csch}^{2}\left(\frac{v}{2}\right)\right) .
$$

Since the function $x \operatorname{csch} x$ is strictly positive and decreasing on $(0, \infty)$, it follows that

$$
\frac{\partial \lambda(v, r)}{\partial v} \begin{cases}<0 & \text { if } r>1 \\ >0 & \text { if } 0<r<1\end{cases}
$$

Accordingly, the function $\lambda(v, r)$ is increasing for $0<r<1$ and decreasing for $r>1$ on $(0, \infty)$. Using L'Hôpital's rule yields

$$
\lim _{v \rightarrow 0^{+}} \lambda(v, r)=\lim _{v \rightarrow 0^{+}} \frac{e^{v}\left(2 \tanh (r v / 2)+r\left(\operatorname{sech}^{2}(r v / 2)-1\right)\right)}{2 e^{v} \tanh (r v / 2)+r\left(e^{v}-1\right) \operatorname{sech}^{2}(r v / 2)}=\frac{1}{2} .
$$

It is easy to see that

$$
\lim _{v \rightarrow \infty} \lambda(v, r)=1-\frac{|t-s|}{2} .
$$

Since the function $\lambda(v, r)$ is even with respect to the variable $r \in \mathbb{R}$ with $r \neq 0$, for $0<|t-s|<1$,

$$
\frac{1}{2}<\lambda(v, s-t)<1-\frac{|t-s|}{2} .
$$

Inequality (2.2) is reversed for $|t-s|>1$. Consequently:

(1) the function $\delta_{s, t ; \lambda}(x)$ is completely monotonic on $(-\alpha, \infty)$ if either $\lambda \leq \frac{1}{2}$ and $0<|t-s|<1$ or $\lambda<1-\frac{1}{2}|t-s|$ and $|t-s|>1$;

(2) the function $-\delta_{s, t ; \lambda}(x)$ is completely monotonic on $(-\alpha, \infty)$ if either $\lambda \geq 1-$ $\frac{1}{2}|t-s|$ and $0<|t-s|<1$ or $\lambda \geq \frac{1}{2}$ and $|t-s|>1$.

Conversely, if the function $\delta_{s, t ; \lambda}(x)$ is completely monotonic, then $\delta_{s, t ; \lambda}(x) \geq 0$ on $(-\alpha, \infty)$, which can be rearranged as

$$
\lambda \leq(x+s)(x+t)\left(\frac{1}{t-s} \int_{s}^{t} \psi^{\prime}(x+u) d u-\frac{1}{2}\left(\frac{1}{x+s}+\frac{1}{x+t}\right)\right) \triangleq \lambda_{s, t}(x) .
$$

From the proof of [8, p. 1981, Lemma 2.4],

$$
\frac{1}{2 x^{2}}-\frac{1}{6 x^{3}}<\frac{1}{x}-\psi^{\prime}(x+1)<\frac{1}{2 x^{2}}-\frac{1}{6 x^{3}}+\frac{1}{30 x^{5}}
$$


for $x>0$. From the left-hand inequality in (2.3) it follows that

$$
\begin{aligned}
\lambda_{s, t}(x)< & (x+s)(x+t) \\
& \quad \times\left(\frac{1}{t-s} \int_{s}^{t}\left(\frac{1}{x+u}+\frac{1}{2(x+u)^{2}}+\frac{1}{6(x+u)^{3}}\right) d u-\frac{1}{2}\left(\frac{1}{x+s}+\frac{1}{x+t}\right)\right) \\
= & \frac{(x+s)(x+t)}{t-s} \log \frac{x+s}{x+t}-x+\frac{1}{2}-\frac{s+t}{2}+\frac{(x+t)^{2}-(x+s)^{2}}{12(t-s)(x+s)(x+t)} \rightarrow \frac{1}{2}
\end{aligned}
$$

as $x \rightarrow \infty$. Similarly, using the right-hand inequality in (2.3) yields

$$
\begin{aligned}
\lambda_{s, t}(x)>(x & +s)(x+t)\left(\frac{1}{t-s} \int_{s}^{t}\left(\frac{1}{x+u}+\frac{1}{2(x+u)^{2}}+\frac{1}{6(x+u)^{3}}-\frac{1}{30(x+u)^{5}}\right) d u\right. \\
& \left.-\frac{1}{2}\left(\frac{1}{x+s}+\frac{1}{x+t}\right)\right) \rightarrow \frac{1}{2}
\end{aligned}
$$

as $x \rightarrow \infty$. As a result, we have the limit

$$
\lim _{x \rightarrow \infty} \lambda_{s, t}(x)=\frac{1}{2}
$$

Since $x \psi(x)=x \psi(x+1)-1$,

$$
\lim _{x \rightarrow 0^{+}}(x \psi(x))=-1 .
$$

From this it follows using a standard argument that

$$
\lim _{x \rightarrow(-\alpha)^{+}} \lambda_{s, t}(x)=1-\frac{|s-t|}{2} .
$$

Therefore, if $|t-s|<1$, then

$$
\lambda \leq \lim _{x \rightarrow \infty} \lambda_{s, t}(x)=\frac{1}{2}<1-\frac{|s-t|}{2}=\lim _{x \rightarrow(-\alpha)^{+}} \lambda_{s, t}(x)
$$

and if $|t-s|>1$, then

$$
\lambda \leq \lim _{x \rightarrow(-\alpha)^{+}} \lambda_{s, t}(x)=1-\frac{|s-t|}{2}<\lim _{x \rightarrow \infty} \lambda_{s, t}(x)=\frac{1}{2} .
$$

The necessity for the function $-\delta_{s, t ; \lambda}(x)$ to be completely monotonic can be similarly reasoned by repeating the above procedure. The proof of Theorem 1.1 is complete.

Proof of Theorem 1.2. This follows from the fact that $\left(\log H_{s, t ; \lambda}(x)\right)^{\prime}=\delta_{s, t ; \lambda}(x)$, as defined in Theorem 1.1, and the definition of logarithmically completely monotonic functions.

Proof of Theorem 1.3. The double inequalities in (1.6) may be deduced readily from the complete monotonicity of the function $\delta_{0,0 ; \lambda}(x)$ shown in Theorem 1.1. 
With the help of the complete monotonicity of the function $\delta_{s, t ; \lambda}(x)$ for $s \neq t$, it easily follows that the double inequality

$$
\begin{aligned}
\beta_{2} \frac{(k-1) !}{t-s}\left(\frac{1}{(x+s)^{k}}-\frac{1}{(x+t)^{k}}\right) & <\frac{(-1)^{k-1}\left(\psi^{(k-1)}(x+t)-\psi^{(k-1)}(x+s)\right)}{t-s} \\
& -\frac{(k-1) !}{2}\left(\frac{1}{(x+s)^{k}}+\frac{1}{(x+t)^{k}}\right) \\
& <\gamma_{2} \frac{(k-1) !}{t-s}\left(\frac{1}{(x+s)^{k}}-\frac{1}{(x+t)^{k}}\right)
\end{aligned}
$$

holds on $(-\alpha, \infty)$ if and only if $\beta_{2} \leq \frac{1}{2}$ and $\gamma_{2} \geq 1-\frac{1}{2}|s-t|$ for $0<|t-s|<1$, and that the double inequality (2.4) is reversed if and only if $\beta_{2} \leq 1-\frac{1}{2}|s-t|$ and $\gamma_{2} \geq \frac{1}{2}$ for $|t-s|>1$. Setting $x+s$ as $a$ and $x+t$ as $b$ in (2.4) produces (1.7).

As early as in 1948, by applying Hölder's integral inequality to the definition (1.1) of the gamma function $\Gamma(x)$ and using the well-known formula $\Gamma(x+1)=x \Gamma(x)$, it was established in [13] that the classical asymptotic relation

$$
\lim _{x \rightarrow \infty} \frac{\Gamma(x+s)}{x^{s} \Gamma(x)}=1
$$

holds for real $s$ and $x$. This implies that

$$
H_{s, t ; \lambda}(x)=\frac{(x+t)^{\lambda /(t-s)}}{(x+s)^{\lambda /(t-s)}} \cdot \frac{x}{\sqrt{(x+s)(x+t)}}\left(x^{s-t} \frac{\Gamma(x+t)}{\Gamma(x+s)}\right)^{1 /(t-s)} \rightarrow 1
$$

as $x \rightarrow \infty$ for $s \neq t$. By virtue of Theorem 1.2, when $0<|t-s|<1$, the function $H_{s, t ; \lambda}(x)$ is decreasing on $(-\alpha, \infty)$ if and only if $\lambda \geq 1-\frac{1}{2}|t-s|$ and it is increasing on $(-\alpha, \infty)$ if and only if $\lambda \leq \frac{1}{2}$. Hence, employing the limit (2.5) implies that the inequality

$$
\frac{(x+t)^{\lambda /(t-s)-1 / 2}}{(x+s)^{\lambda /(t-s)+1 / 2}}\left(\frac{\Gamma(x+t)}{\Gamma(x+s)}\right)^{1 /(t-s)}>1
$$

holds on $(-\alpha, \infty)$ if and only if $\lambda \geq 1-\frac{1}{2}|t-s|$ and reverses on $(-\alpha, \infty)$ if and only if $\lambda \leq \frac{1}{2}$. Similarly, when $|t-s|>1$, the function $H_{s, t ; \lambda}(x)$ is decreasing on $(-\alpha, \infty)$ if and only if $\lambda \geq \frac{1}{2}$ and it is increasing on $(-\alpha, \infty)$ if and only if $\lambda \leq 1-\frac{1}{2}|t-s|$. This leads to the conclusion that the reversed version of inequality (2.6) is valid. Finally, the double inequality

$$
\frac{(x+s)^{\beta_{3} /(t-s)+1 / 2}}{(x+t)^{\beta_{3} /(t-s)-1 / 2}}<\left(\frac{\Gamma(x+t)}{\Gamma(x+s)}\right)^{1 /(t-s)}<\frac{(x+s)^{\gamma_{3} /(t-s)+1 / 2}}{(x+t)^{\gamma_{3} /(t-s)-1 / 2}}
$$

holds on $(-\alpha, \infty)$ if and only if $\beta_{3} \geq 1-\frac{1}{2}|t-s|$ and $\gamma_{3} \leq \frac{1}{2}$ for $0<|t-s|<1$; the double inequality (2.7) is reversed if and only if $\beta_{3} \leq 1-\frac{1}{2}|t-s|$ and $\gamma_{3} \geq \frac{1}{2}$ for $|t-s|>1$. Furthermore, replacing $x+s$ by $a$ and $x+t$ by $b$ reproduces (1.8). 
The double inequality (1.9) comes from the fact that the inequality $H_{s, t ; \lambda}(\rho)>$ $H_{s, t, \lambda}(x)$ holds on $(\rho, \infty)$ if and only if either $\lambda \geq 1-\frac{1}{2}|t-s|$ for $0<|t-s|<1$ or $\lambda \geq \frac{1}{2}$ and $|t-s| \geq 1$, and that it is reversed if and only if either $\lambda \leq \frac{1}{2}$ for $0<|t-s|<1$ or $\lambda \leq 1-\frac{1}{2}|t-s|$ and $|t-s| \geq 1$. The proof of Theorem 1.3 is complete.

\section{Acknowledgements}

This paper was completed during the first author's visit to the Research Group in Mathematical Inequalities and Applications (RGMIA), School of Engineering and Science, Victoria University, Australia, between March 2008 and February 2009, with a grant from the China Scholarship Council. The first author would like to express his sincere appreciation to RGMIA colleagues for their invitation and hospitality throughout this period. The authors would like to thank the anonymous referees for helpful corrections to and valuable comments on the original version of this paper.

\section{References}

[1] C. Berg, 'Integral representation of some functions related to the gamma function', Mediterr. $J$. Math. 1(4) (2004), 433-439.

[2] C.-P. Chen and F. Qi, 'Logarithmically completely monotonic functions relating to the gamma function', J. Math. Anal. Appl. 321 (2006), 405-411.

[3] N. Elezović, C. Giordano and J. Pečarić, 'The best bounds in Gautschi's inequality', Math. Inequal. Appl. 3 (2000), 239-252.

[4] R. A. Horn, 'On infinitely divisible matrices, kernels and functions', Z. Wahrscheinlichkeitstheorie Verw. Geb 8 (1967), 219-230.

[5] F. Qi, 'A completely monotonic function involving the divided difference of the psi function and an equivalent inequality involving sums', ANZIAM J. 48(4) (2007), 523-532.

[6] F. Qi, 'Three classes of logarithmically completely monotonic functions involving gamma and psi functions', Integral Transforms Spec. Funct. 18(7) (2007), 503-509.

[7] F. Qi, 'Bounds for the ratio of two gamma functions', J. Inequal. Appl. 2010 (2010), Article ID 493058.

[8] F. Qi and B.-N. Guo, 'Completely monotonic functions involving divided differences of the di- and tri-gamma functions and some applications', Commun. Pure Appl. Anal. 8(6) (2009), 1975-1989.

[9] F. Qi and Q.-M. Luo, 'Bounds for the ratio of two gamma functions-from Wendel's and related inequalities to logarithmically completely monotonic functions', Banach J. Math. Anal. 6(2) (2012), 132-158.

[10] F. Qi, Q.-M. Luo and B.-N. Guo, 'Complete monotonicity of a function involving the divided difference of digamma functions', Sci. China Math. 56 (2013), in press.

[11] R. L. Schilling, R. Song and Z. Vondraček, Bernstein Functions, de Gruyter Studies in Mathematics, 37 (De Gruyter, Berlin, 2010).

[12] G. N. Watson, 'A note on gamma functions', Proc. Edinb. Math. Soc. 11(2) (1958/1959), Edinburgh Math Notes (42) (misprinted (41)) (1959), 7-9.

[13] J. G. Wendel, 'Note on the gamma function', Amer. Math. Monthly 55(9) (1948), 563-564.

[14] D. V. Widder, The Laplace Transform (Princeton University Press, Princeton, NJ, 1946).

FENG QI, College of Mathematics, Inner Mongolia University for Nationalities, Tongliao City 028043, Inner Mongolia Autonomous Region, PR China e-mail: qifeng618@gmail.com, qifeng618@hotmail.com, qifeng618@qq.com 
PIETRO CERONE, Department of Mathematics and Statistics, La Trobe University, Bundoora, Victoria 3086, Australia e-mail: pcerone53@gmail.com,p.cerone@latrobe.edu.au

SEVER S. DRAGOMIR, School of Engineering and Science, Victoria University, PO Box 14428, Melbourne, Victoria 8001, Australia and

School of Computational and Applied Mathematics, University of the Witwatersrand, Johannesburg, Private Bag 3, Wits 2050, South Africa e-mail: sever.dragomir@vu.edu.au 